\title{
Relationship between jaw movement and masticatory performance in adults with natural dentition
}

\author{
ELAN IGNACIO FLORES-OROZCO ${ }^{1,2}$, BERNAT ROVIRA-LASTRA $^{1}$, EVA WILLAERT ${ }^{1}$, \\ MARIA PERAIRE $^{1} \&$ JORDI MARTINEZ-GOMIS ${ }^{1}$
}

${ }^{1}$ Department of Prosthodontics, Faculty of Dentistry, University of Barcelona, Barcelona, Spain, and ${ }^{2}$ Department of Prosthodontics, Faculty of Dentistry, Autonomous University of Nayarit, Tepic, Nayarit, México

\begin{abstract}
Objective. This study determines the relationship between several characteristics of jaw movement and masticatory performance determined by multiple regression analysis and adjusted for occlusal contact area and bite force. Materials and methods. Forty-two young adults with natural dentition participated in this cross-sectional study. Occlusal contact area was determined at the maximum intercuspal position by scanning interocclusal records. Maximum unilateral force was measured by means of a gnathodynamometer. The height and amplitude of mastication, occlusal glide length, lateral guidance angle, anterior-posterior distance and cycle duration were recorded using the ARCUSdigma II system. Masticatory performance was determined by sieving the Optosil particles resulting from 20 chewing cycles. Results. Median particle size was negatively associated with height of mastication, maximum bite force, occlusal contact area and amplitude of mastication. Stepwise multiple linear regression analysis revealed that the height of mastication and dental guidance angle are the characteristics of jaw movement most closely related to masticatory performance. Conclusions. In adults with natural dentition, a large vertical height of mastication and a small dental guidance angle are the characteristics of jaw movement most closely associated with good masticatory performance, determined by multiple regression analysis and adjusted for occlusal contact area and bite force.
\end{abstract}

Key Words: Chewing, mandibular movement, masticatory efficiency, bagged silicone, kinesiography

\section{Introduction}

Prosthodontic treatment aims to restore or improve masticatory function [1]. Masticatory function is evaluated by self-assessment of chewing ability and/or objective masticatory performance assessed in laboratory tests [2]. Masticatory performance can be determined by quantifying the degree of fragmentation of a test food after a fixed number of chewing cycles [3]. Although natural foods such as almonds have been used as a test food [4-7], silicone is considered more appropriate to assess masticatory function [8]. Therefore, the most recent studies use silicone to assess masticatory function [9-16]; silicon pieces in a latex bag has been demonstrated to be a reliable method [17].

Variations on masticatory function may be due to extrinsic factors related to the food and/or intrinsic factors related to the individual characteristics [18]. Among the intrinsic factors, occlusal contact area in the intercuspal position (ICP) and bite force are the key factors in masticatory performance $[7,9,12,19]$. Other factors that can affect masticatory performance include body size [9], age [11], sex [7], salivary flow rate [19], temporomandibular disorders [20] and jaw movements $[4-6,10,15,16]$. However, which characteristics of masticatory mandibular movement are most closely related to masticatory performance is controversial $[5,6,15,16]$.

The inter-relationships between some of the intrinsic factors $[14,21]$ means that masticatory performance should be studied using multivariate techniques [7]. Using multiple regression analysis it has been reported that vertical amplitude, closing duration, closing angle and maximum bite force emerge as significant predictors of the Mixing Ability 
Index [22]. Although mixing ability may be comparable with masticatory performance in subjects with limited masticatory performance, the comminution test is better suited to discriminate masticatory performance in subjects who suffer no such imitation [23]. To the best of our knowledge, no studies to date have used multivariate analysis to relate mandibular movement variables with masticatory performance using a comminution test.

The study reported here aimed to determine the relationship between several characteristics of jaw movement and masticatory performance determined by multiple regression analysis and adjusted for occlusal contact area and bite force, in young adults with natural dentition. The null hypothesis tested was that jaw movement parameters are not related with masticatory performance adjusted for occlusal contact area and bite force.

\section{Materials and methods}

In this cross-sectional study, 42 adults (23 women, mean age $=27$ years) with natural dentition were selected from volunteer students and staff who had participated in a previous study [17]. Subjects with fewer than 24 natural teeth, those undergoing active orthodontic treatment and those suffering orofacial pain were excluded. Among the participants, 31 had Angle class I bilateral and 11 had unilateral or bilateral class II. No subject had severe malocclusion or temporomandibular disorders that could affect mandibular movement. The study was approved by the Ethics Committee of the Barcelona University Dental Hospital (Code 17/12) and all the experiments were carried out in accordance with the principles of the Helsinki Declaration [24].

Occlusal contact area in the ICP was measured using bite registration material (Occlufast Rock; Zhermack, Badia Polesine, Italy), which was applied to all the mandibular teeth, and subjects were asked to bite down firmly into the ICP for $1 \mathrm{~min}$, until the material had set. The occlusal registration was trimmed, scanned and analyzed by means of computer software and considering occlusal contact as an interocclusal distance of $200 \mu \mathrm{m}$ or less [12,13]. A bite-force transducer (gnathodynamometer; Technical University of Catalonia, Barcelona, Spain) was used to measure unilateral forces. This device was calibrated with loads from $0-1200 \mathrm{~N}$ by means of a compression test machine at the Department of Materials Science and Metallurgy of the Technical University of Catalonia. Maximum bite force was measured three times between the first molars on the right and left sides and the highest value was selected $[12,13]$.

Masticatory jaw movements were recorded using the ARCUSdigma II system (KaVo, Biberach, Germany). The ARCUSdigma measuring bow was placed around each subject's head and the
ARCUSdigma transmitter was affixed to the mandibular arch using the mandibular attachment, which had previously been adapted to the labial surfaces of the anterior mandibular teeth by acrylic (Trim, Bosworth Company, Skokie, IL) and fixed using cyanoacrylate. The subjects were asked to perform right- and leftsided lateral guidance movements with the teeth in light contact, starting and ending in the ICP. Afterwards, they were asked to chew naturally, without imposing any side of mastication, a test medium known as 'bagged silicone' for 20 cycles in order to comminute the pieces [17]. Optosil tablets (5 mm thick, $20 \mathrm{~mm}$ diameter) (Optosil P Plus; Heraeus Kulzer, Hanau, Germany) were produced as described by Albert et al. [25], cut into quarters, and three quarters placed in a latex bag which was sealed with cyanoacrylate adhesive. The masticatory assay was repeated four more times and the particles from the five assays $(10 \mathrm{~g})$ were passed through a series of eight sieves $(0.25,0.425,0.85,2,2.8,3.15$, 4 and $5.6 \mathrm{~mm}$ ) while being shaken for $1 \mathrm{~min}$. After the cumulative weight distribution of the sieve contents had been determined, median particle size was calculated for each subject using the Rosin-Rammler equation $\left[Q_{w}(X)=1-2 E-\left(X / X_{50}\right)^{b}\right]$, where $Q_{w}(X)$ is the fraction of particles by weight with a diameter smaller than $X$, the median particle size $\left(X_{50}\right)$ is the size of a theoretical sieve through which $50 \%$ of the weight can pass and $b$ describes the breadth of the particle size distribution [26]. Therefore, the lower the median particle size value, the better the masticatory performance. Characteristics of jaw movement such as height and amplitude of mastication, occlusal glide length, lateral guidance angle, anterior-posterior distance and cycle duration were obtained as the mean of the 100 masticatory cycles (Figure 1).

The normal distribution of the data was confirmed by means of a Kolmogorov-Smirnov test. Pearson correlation coefficients were calculated to evaluate the bivariate correlation between the variables and median particle size. Finally, and because the variables are inter-related, stepwise multiple linear regression analysis was performed to determine whether the variables contribute significantly to explaining the median particle size (IBM SPSS Statistics, version 21.0, Chicago, IL). $p$-values below 0.05 were considered significant.

\section{Results}

The Pearson correlation coefficients between median particle size and variables related to mandibular movement, maximum bite force and occlusal contact area are shown in Table I. Median particle size was negatively associated with the height of mastication, maximum bite force, occlusal contact area and the amplitude of mastication. Stepwise multiple regression analysis showed that the height of mastication, 


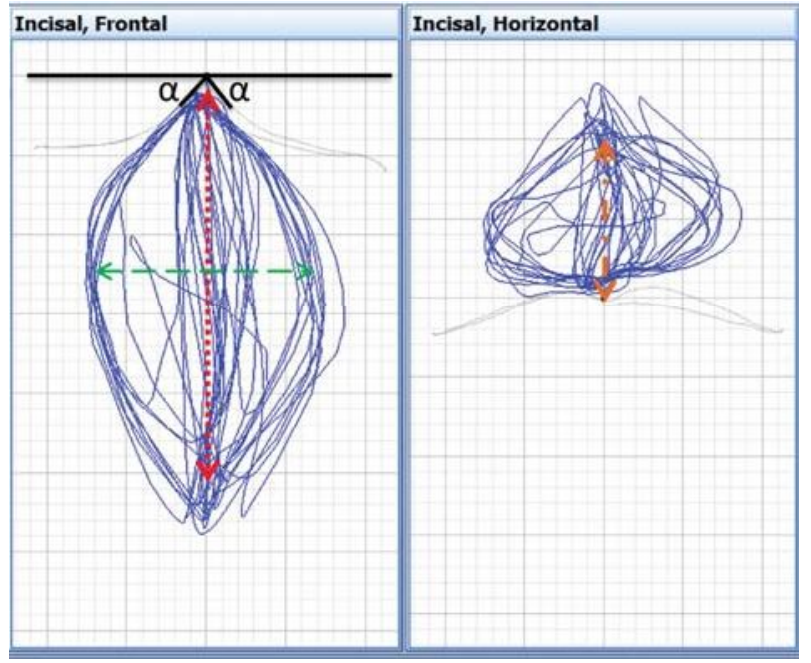

Figure 1. Characteristics of jaw movement. For the frontal plane, the height of mastication was measured from the intercuspal position (ICP) to the maximum opening (short dashed red line); the amplitude of mastication was obtained as the maximum lateral displacement of the jaw (long dashed green line); the occlusal glide length was measured as the lateral displacement between ICP and the point in the closing phase at $0.5 \mathrm{~mm}$ from occlusal contact (plain yellow line); and the lateral guidance angle $(\alpha)$ was measured as the angle between the horizontal plane and the line connecting the ICP and a point located $1.5 \mathrm{~mm}$ away in lateral displacement. For the horizontal plane, the anterior-posterior distance was also calculated (dash-dot-dash orange line).

maximum bite force and lateral guidance angle were the most important factors affecting the median particle size (Table II). These three variables accounted for $55 \%$ of the variation in masticatory performance.

\section{Discussion}

Among all the variables studied, the height of mastication cycles correlated most closely with masticatory performance, explaining $34 \%$ of its variation and, therefore, the null hypothesis was rejected. Bite force and lateral guidance angle explained an additional $22 \%$ of the variation in masticatory performance. This means that improved mastication was associated with high vertical mastication, great bite force, increased occlusal contact area and a large mandibular amplitude during mastication. Lepley et al. [15] report that better masticatory performers have a shorter opening period than poorer performers, but similar vertical excursion. Furthermore, improved mastication has been associated with a faster chewing rate [5,6], increased mandibular velocity [6] and a low lateral guidance angle [5]. Since the majority of these characteristics of jaw movements are inter-related (e.g. amplitude and height of mastication; cycle duration and lateral guidance angle; occlusal glide length and amplitude) [5] these apparent discrepancies between studies may be due to the differences in the populations and may not actually reflect different associations.

Although the occlusal contact area was significantly associated with masticatory performance, this association lost its significance after adjusting for bite force and jaw movement parameters in the multiple linear regression analysis. Therefore, bite force and mandibular movement seem to be more directly related to masticatory performance than the occlusal contact area is, in a population with a full or nearly full complement of natural teeth. When fixed prosthodontic restoration is required to replace lost occlusal surfaces or missing teeth, the occlusal scheme should take these findings into account.

These results are in agreement with another study that found that vertical amplitude, closing duration, closing angle and maximum bite force are significant predictors of mixing ability, accounting for $63 \%$ of inter-subject variation in the Mixing Ability Index [22]. Whereas the mixing ability test evaluates the ability to mix a food bolus, median particle size evaluates the ability to comminute food. The coincidence of the results means that bite force, vertical amplitude and lateral guidance angle are the key predictors of two different features of the chewing process [23].

One limitation of the present study is that the use of the ARCUSdigma system may interfere with the natural and semi-automatic act of mastication. However,

Table I. Pearson's correlation coefficient matrix of the masticatory parameters.

\begin{tabular}{|c|c|c|c|c|c|c|c|c|c|}
\hline & MPS & $\mathrm{BF}$ & OCA & Amp & $\mathrm{H}$ & A-P & OGL & LGA & $\mathrm{CD}$ \\
\hline Median particle size (MPS) (mm) & 1 & & & & & & & & \\
\hline Bite force $(\mathrm{BF})(\mathrm{N})$ & $-0.56 \star \star \star$ & 1 & & & & & & & \\
\hline Occlusal contact area (OCA) $\left(\mathrm{mm}^{2}\right)$ & $-0.31 \star$ & 0.20 & 1 & & & & & & \\
\hline Amplitude (Amp) (mm) & -0.26 * & -0.07 & 0.09 & 1 & & & & & \\
\hline Height $(\mathrm{H})(\mathrm{mm})$ & $-0.60 \star \star \star$ & 0.26 * & 0.16 & $0.48^{\star \star \star}$ & 1 & & & & \\
\hline Anterior-posterior distance (A-P) (mm) & 0.10 & -0.04 & 0.07 & -0.04 & 0.21 & 1 & & & \\
\hline Occlusal glide length (OGL) (mm) & -0.11 & -0.02 & 0.19 & $0.59 \star \star \star$ & 0.24 & -0.04 & 1 & & \\
\hline Lateral guidance angle (LGA) $(\stackrel{\circ}{)})$ & 0.17 & 0.18 & 0.04 & $-0.40 \star \star$ & -0.01 & -0.19 & -0.05 & 1 & \\
\hline Cycle duration (CD) (ms) & -0.12 & -0.05 & $0.33^{\star}$ & -0.07 & 0.25 & 0.13 & $0.27^{\star}$ & 0.01 & 1 \\
\hline
\end{tabular}

${ }^{\star} p<0.05 ;{ }^{\star \star} p<0.01 ;{ }^{\star \star \star} p<0.001$. 
Table II. Stepwise regression models of factors related to median particle size.

\begin{tabular}{llllll}
\hline Model & Variables included & Beta & $R$ & $R_{a}^{2}$ & $F$ (Sig.) \\
\hline 1 & Height of mastication (mm) & -0.466 & 0.60 & 0.34 & $22.0(<0.001)$ \\
2 & Bite force (N) & -0.478 & 0.73 & 0.50 & $21.7(<0.001)$ \\
3 & Lateral guidance angle (⿳) & 0.247 & 0.77 & 0.55 & $17.9(<0.001)$ \\
\hline
\end{tabular}

$R_{a}^{2}$, adjusted $R^{2}$ (\% variance explained); $F$ (Sig.), $F$-value and significance.

the high precision of the device and the simultaneous recording of masticatory performance and masticatory movements justify its use. Because only one test food was used to assess masticatory function, the results are, thus, only applicable to this type of food. However, a high level of concordance was found between chewing bagged or unbagged silicon on masticatory laterality, masticatory performance and chewing cycle duration. Recorded maximum bite force may not correspond to actual maximum muscular potential because of the increase of interincisal distance of $\sim 20 \mathrm{~mm}$. However, this was similar for all the subjects and these values can be useful in correlating with masticatory performance.

In conclusion, in adults with natural dentition, a large vertical height of mastication and a small dental guidance angle are the characteristics of jaw movement most closely associated with good masticatory performance, determined by multiple regression analysis and adjusted for occlusal contact area and bite force.

\section{Acknowledgements}

The authors would like to thank the Language Services of the University of Barcelona for correcting our English. This research received partial funding from the Bellvitge Campus Research Committee, University of Barcelona (ACESB 08/04) and from the Faculty of Dentistry, University of Barcelona.

AQ4 Declaration of interest: The authors report no conflicts of interest. The authors alone are responsible for the content and writing of the paper.

\section{References}

[1] Jokstad A, Orstavik J, Ramstad T. A definition of prosthetic dentistry. Int J Prosthodont 1998;11:295-301.

[2] Feine JS, Lund JP. Measuring chewing ability in randomized controlled trials with edentulous populations wearing implant prostheses. J Oral Rehabil 2006;33:301-8.

[3] van der Bilt A, Fontijn-Tekamp FA. Comparison of single and multiple sieve methods for the determination of masticatory performance. Arch Oral Biol 2004;49:155-60.

[4] Helkimo E, Carlsson GE, Helkimo M. Chewing efficiency and state of dentition. A methodologic study. Acta Odontol Scand 1978;36:33-41.
[5] Wilding RJ, Lewin A. The determination of optimal human jaw movements based on their association with chewing performance. Arch Oral Biol 1994;39:333-43.

[6] Ow RK, Carlsson GE, Karlsson S. Relationship of masticatory mandibular movements to masticatory performance of dentate adults: A method study. J Oral Rehabil 1998;25:821-9.

[7] Hatch JP, Shinkai RS, Sakai S, Rugh JD, Paunovich ED. Determinants of masticatory performance in dentate adults. Arch Oral Biol 2001;46:641-8.

[8] Edlund J, Lamm CJ. Masticatory efficiency. J Oral Rehabil 1980;7:123-30.

[9] Julien KC, Buschang PH, Throckmorton GS, Dechow PC. Normal masticatory performance in young adults and children. Arch Oral Biol 1996;41:69-75.

[10] Buschang PH, Throckmorton GS, Travers KH, Johnson G. The effects of bolus size and chewing rate on masticatory performance with artificial test foods. J Oral Rehabil 1997;24: $522-6$.

[11] Toro A, Buschang PH, Throckmorton G, Roldan S. Masticatory performance in children and adolescents with Class I and II malocclusions. Eur J Orthod 2006;28:112-19.

[12] Lujan-Climent M, Martinez-Gomis J, Palau S, Ayuso-Montero R, Salsench J, Peraire M. Influence of static and dynamic occlusal characteristics and muscle force on masticatory performance in dentate adults. Eur J Oral Sci 2008;116: 229-36.

[13] Martinez-Gomis J, Lujan-Climent M, Palau S, Bizar J, Salsench J, Peraire M. Relationship between chewing side preference and handedness and lateral asymmetry of peripheral factors. Arch Oral Biol 2009;54:101-7.

[14] Gomes SG, Custodio W, Faot F, Del Bel Cury AA, Garcia RC. Masticatory features, EMG activity and muscle effort of subjects with different facial patterns. J Oral Rehabil 2010;37:813-19.

[15] Lepley C, Throckmorton G, Parker S, Buschang PH. Masticatory performance and chewing cycle kinematics-are they related? Angle Orthod 2010;80:295-301.

[16] Sánchez-Ayala A, Farias-Neto A, Campanha NH, Garcia RC. Relationship between chewing rate and masticatory performance. Cranio 2013;31:118-22.

[17] Rovira-Lastra B, Flores-Orozco EI, Salsench J, Peraire M, Martinez-Gomis J. Is the side with the best masticatory performance selected for chewing? Arch Oral Biol 2014;59: 1316-20.

[18] Woda A, Foster K, Mishellany A, Peyron MA. Adaptation of healthy mastication to factors pertaining to the individual or to the food. Physiol Behav 2006;89:28-35.

[19] Ikebe K, Matsuda K, Morii K, Furuya-Yoshinaka M, Nokubi T, Renner RP. Association of masticatory performance with age, posterior occlusal contacts, occlusal force, and salivary flow in older adults. Int J Prosthodont 2006;19: 475-81.

[20] Peroz I, Tai S. Masticatory performance in patients with anterior disk displacement without reduction in comparison with symptom-free volunteers. Eur J Oral Sci 2002;110: $341-4$. 
[21] Salsench J, Martínez-Gomis J, Torrent J, Bizar J, Samsó J, Peraire M. Relationship between duration of unilateral masticatory cycles and the type of lateral dental guidance: A preliminary study. Int J Prosthodont 2005;18:339-46.

[22] Yoshida E, Fueki K, Igarashi Y. Association between food mixing ability and mandibular movements during chewing of a wax cube. J Oral Rehabil 2007;34:791-9.

[23] van der Bilt A, Mojet J, Tekamp FA, Abbink JH. Comparing masticatory performance and mixing ability. J Oral Rehabil 2010;37:79-84.
[24] World Medical Association. World Medical Association Declaration of Helsinki: Ethical principles for medical research involving human subjects. JAMA 2013;310: 2191-4.

[25] Albert TE, Buschang PH, Throckmorton GS. Masticatory performance: A protocol for standardized production of an artificial test food. J Oral Rehabil 2003;30:720-2.

[26] Olthoff LW, van der Bilt A, Bosman F, Kleizen HH. Distribution of particle sizes in food comminuted by human mastication. Arch Oral Biol 1984;29:899-903.

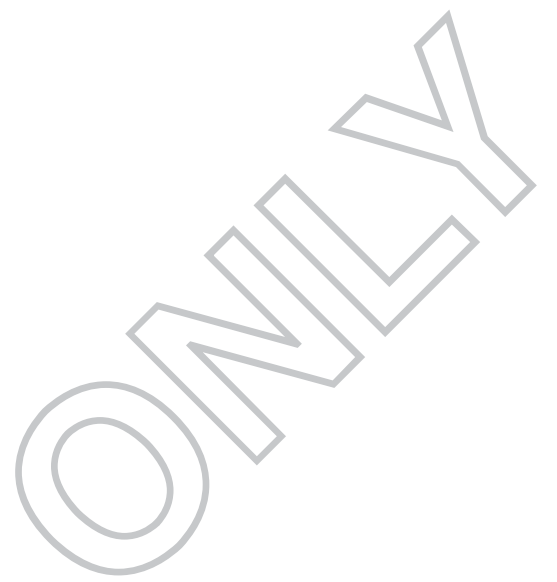

